\title{
Understanding nuclear "pasta": current status and future prospects
}

\author{
Gentaro Watanabe ${ }^{\dagger}$ \\ NORDITA, Blegdamsvej 17, DK-2100 Copenhagen Ø, Denmark \\ The Institute of Chemical and Physical Research (RIKEN), Saitama 351-0198, Japan
}

\begin{abstract}
In cores of supernovae and crusts of neutron stars, nuclei can adopt interesting shapes, such as rods or slabs, etc., which are referred to as nuclear "pasta." In recent years, we have studied the pasta phases focusing on their dynamical aspects with a quantum molecular dynamic (QMD) approach. In this article, we review these works. We also focus on the treatment of the Coulomb interaction.
\end{abstract}

\section{INTRODUCTION}

In ordinary matter on the earth, atomic nuclei are roughly spherical. This fact can be understood in the liquid drop picture of the nucleus as being a result of the surface tension of nuclear matter, which favors a spherical nucleus, being greater than the Coulomb repulsion between protons, which tends to make the nucleus deform. However, in supernova cores and neutron stars, the situation changes completely (see, e.g., Refs. [1, 2]): when the density of matter approaches that of atomic nuclei, i.e., the normal nuclear density $\rho_{0}$, nuclei are closely packed and the effect of the electrostatic energy becomes comparable to that of the surface energy. Consequently, around a density $\rho \simeq \rho_{0} / 2$, the energetically favorable configuration could be rod-like or slab-like nuclei embedded in the gas phase, or rod-like or spherical bubbles of the gas phase embedded in nuclear matter [3, 4]. Such phases with exotic shapes of nuclei are referred to as nuclear "pasta" phases.

Properties of the pasta phases in equilibrium states have been investigated by many authors so far [5, 6, 7, 8, 9, 10]. These earlier works have confirmed that, for various nuclear models, the nuclear shape changes in the sequence sphere, cylinder, slab, cylindrical hole, spherical hole, uniform with increasing density. This conclusion holds for the case of non-zero temperatures with a constant entropy [6] as well as for the zero temperature case. However, in these works (except for Ref. [5]) they took account of only several specific nuclear shapes and determined the energetically favorable one by calculating the energy for these assumed structures within a liquid drop model or the Thomas-Fermi approximation. Thus the phase diagram at subnuclear densities and the existence of the pasta phases should be examined without assuming the nuclear shape. It is also noted that at typical temperatures of the collapsing cores, several $\mathrm{MeV}$, effects of thermal fluctuations on the nucleon distribution are significant. However, these thermal fluctuations cannot be described properly by mean-field theories such as the Thomas- 
Fermi and the Hartree-Fock approximations.

In the processes of supernova explosion and succeeding neutron star formation, the pasta phases can be formed in two stages. During the gravitational collapse, matter in the collapsing core is compressed and the central region starts to solidify into a bcc lattice because the Coulomb repulsion among nuclei gets stronger. In the later stage of the collapse, the central density reaches $\rho_{0}$ and the pasta phases could be formed from the bcc lattice due to the compression. After a bounce of the core takes place, the temperature of the core increases to $O(10) \mathrm{MeV}$ and nuclei melt completely. As the cooling process of the protoneutron star proceeds later on, the pasta phases as well as normal spherical nuclei could be produced again from hot uniform nuclear matter at subnuclear densities.

The previous studies, which are based on a comparison of the energy with an assumption of the nuclear shape, cannnot answer whether or not the pasta phases can be formed dynamically within the time scale of the neutron star cooling nor whether or not the transitions between them, which is accompanied by drastic changes of nuclear shape, can be realized under nonequilibrium conditions in the collapsing cores. To solve these problems, molecular dynamic methods for nucleon many-body systems are suitable. They treat the motion of the nucleonic degrees of freedom and can describe thermal fluctuations and many-body correlations beyond the mean-field level.

Using one of the molecular dynamic methods, the quantum molecular dynamics (QMD) [11], we have arrived at the two following major conclusions [12, 13, 14]. (1) The pasta phases are formed from hot uniform nuclear matter by cooling it down within a time scale of $\sim O\left(10^{3}-10^{4}\right) \mathrm{fm} / c$. This supports the idea that the pasta phases exist in neutron star crusts. (2) The transition from rod-like nuclei to slab-like nuclei and that from slab-like nuclei to rod-like bubbles can be realized by compression of matter. This suggests that the pasta phases could be formed in collapsing cores.

Throughout the present article, we set the Boltzmann constant $k_{\mathrm{B}}=1$.

\section{METHOD: QUANTUM MOLECULAR DYNAMICS}

Among various versions of the molecular dynamic models, QMD [11] is the most practical and suitable for studying the pasta phases. This method is so efficient that we can describe rod-like and slab-like nuclei in terms of nucleon degrees of freedom even though they consist of macroscopic number of nucleons. In addition, we focus on the pasta structure and the configuration of nuclei on a macroscopic scale, where the shell effects [15, 16, 17], which cannot be described by QMD, may be less important (in the case of supernova matter, shell effects are always small). Thus QMD is a good approximation for our present problem.

\section{Model Hamiltonian}

In our studies, we use a nuclear force given by a QMD model Hamiltonian with the medium-equation-of-state parameter set in Ref. [18]. This Hamiltonian contains 
the momentum-dependent "Pauli potential" to reproduce the effects of the Pauli principle phenomenologically. The Pauli potential generates repulsive forces between two identical particles close together in phase space. Parameters in the Pauli potential are determined by fitting the kinetic energy of the free Fermi gas at zero temperature.

This Hamiltonian reproduces the binding energy of symmetric nuclear matter, 16 $\mathrm{MeV}$ per nucleon, at the normal nuclear density $\rho_{0}=0.165 \mathrm{fm}^{-3}$ and other saturation properties: the incompressibility is set to be $280 \mathrm{MeV}$ and the symmetry energy is 34.6 $\mathrm{MeV}$ [18]. This Hamiltonian also well reproduces the properties of stable nuclei relevant for the situations we consider: the binding energy (except for light nuclei from ${ }^{12} \mathrm{C}$ to ${ }^{20} \mathrm{Ne}$ ) [18], and the rms radius of the ground state of heavy nuclei with $A \gtrsim 100$ [19]. It is also confirmed that another QMD Hamiltonian close to this model provides a good description of nuclear reactions including the low energy region (several MeV per nucleon) [20], which is one of the essential elements when one studies the dynamical processes as in Sect. III-2.

\section{Coulomb Interaction and its Screening Effect}

The Coulomb interaction is one of the essential elements for the pasta phases. One must carefully treat the long-range nature of the Coulomb interaction since electron screening is negligibly small in this system [1, 21, 22]. A key quantity in determining the importance of electron screening is the ratio of the scale of the inhomogeneity, typically half the internuclear spacing $R$, to the Thomas-Fermi screening length $\lambda_{\mathrm{TF}}=$ $\left[4 \pi e^{2}\left(\partial n_{e}^{(0)} / \partial \mu_{e}^{(0)}\right)_{n_{e}(0)}\right]^{-1 / 2}=\sqrt{\pi / 4 \pi}\left(k_{e}^{(0)}\right)^{-1}$, where $n_{e}^{(0)}$ and $\mu_{e}^{(0)}$ are the averaged

electron number density and chemical potential, respectively, and $k_{e}^{(0)}=\sqrt{3 \pi^{2} n_{e}^{(0)}}$ is the electron Fermi wave vector. We note that, in the density region of the pasta phases, $R / \lambda_{\mathrm{TF}}<1$ [23, 1], which means that it is a good approximation to assume that the electron density distribution is uniform and neglect the screening effect; but one must take account of the long-range nature of the Coulomb force by, e.g., the Ewald summation procedure [12].

It is fortunate that the screening effect is small; otherwise one has to solve equations of motion for the electronic degrees of freedom together with that for the nucleonic ones because the coupling between these two components cannot be neglected. Thus introducing a screening length shorter than the actual value for calculating the Coulomb force between protons keeping the density of electrons uniform is inconsistent. Let us restrict ourselves to the equilibrium state and discuss this point more quantitatively using a liquid drop model in the Wigner-Seitz approximation. The energy density of a relativistic degenerate electron gas with an inhomogeneous density distribution $n_{e}(\mathbf{r})=n_{e}^{(0)}+\delta n_{e}(\mathbf{r})$ is given by $E_{e}\left[n_{e}(\mathbf{r})\right] \equiv E_{e}^{(0)}\left(n_{e}^{(0)}\right)+\delta E_{e} \simeq E_{e}^{(0)}\left(n_{e}^{(0)}\right)[1+$ $\left.\left(2 / 9 V_{\mathrm{c}}\right) \int_{\text {cell }} d^{3} r\left(\delta n_{e}(\mathbf{r}) / n_{e}^{(0)}\right)^{2}\right]$, with $E_{e}^{(0)}\left(n_{e}^{(0)}\right)=(3 / 4) \hbar c k_{e}^{(0)} n_{e}^{(0)}$, where $V_{\mathrm{c}}$ is the volume of the Wigner-Seitz cell. If the screening effect is significant, the Coulomb energy among protons decreases, but on the other hand, the $\left(\delta n_{e}(\mathbf{r}) / n_{e}^{(0)}\right)^{2}$ term cannot be neglected. Calculations assuming a small $\lambda_{\mathrm{TF}}$ and uniform $n_{e}$ artificially underestimate the Coulomb energy among protons but discard the significant contribution of $\delta E_{e}$. Now we 
estimate $\delta E_{e}$ for an unrealistically small value of $\lambda_{\mathrm{TF}}$. We take the phase with spherical nuclei in the density region where the pasta phases start to appear, i.e., the volume fraction of nuclei is $u=\left(r_{\mathrm{N}} / r_{\mathrm{c}}\right)^{3} \simeq 1 / 8$ according to the condition of the fission instability of spherical nuclei [1]. Here $r_{\mathrm{N}}$ and $r_{\mathrm{c}}$ are the radii of nuclei and the Wigner-Seitz cell, respectively. Taking supernova matter for simplicity, in which the density of dripped neutrons is negligible, with the nucleon density in nuclei $n=0.1 \mathrm{fm}^{-3}$, proton fraction in nuclei $x_{\mathrm{N}}=0.3, r_{\mathrm{c}}=15 \mathrm{fm}$, and $r_{\mathrm{N}}=15 / 2 \mathrm{fm}$, and using expressions in Ref. [21], $\delta E_{e}$ per nucleon, $\delta E_{e} / n_{\mathrm{N}}$, is estimated as $\delta E_{e} / n_{\mathrm{N}} \simeq 0.23 \mathrm{MeV}^{1}$ for $\lambda_{\mathrm{TF}}=10 \mathrm{fm}$ (this value is adopted in Ref. [24]). Note that this is much larger than the energy difference between different pasta phases in the same density region in supernova matter, $O(10)$ $\mathrm{keV}$ per nucleon.

\section{SIMULATIONS AND RESULTS}

In the present section, let us review our previous works [12, 13, 14]. In our QMD simulations, we treated a system which consists of neutrons, protons, and electrons in a cubic box with periodic boundary conditions. The system is not magnetically polarized, i.e., it contains equal numbers of protons (and neutrons) with spin up and spin down. The relativistic degenerate electrons which ensure charge neutrality are regarded as a uniform background [1, 21, 22]. The Coulomb interaction is calculated by the Ewald method taking account of the Gaussian charge distribution of the proton wave packets.

\section{Realization of the Pasta Phases and Equilibrium Phase Diagrams}

In Refs. [12, 13], we showed that the pasta phases are produced from hot uniform nuclear matter by cooling it down and we studied phase diagrams at zero and nonzero temperatures. In these works, we first prepared a uniform hot nucleon gas at a temperature $T \sim 20 \mathrm{MeV}$. We then cooled it down slowly until the temperature got $\sim 0.1$ $\mathrm{MeV}$ or less for $O\left(10^{3}-10^{4}\right) \mathrm{fm} / c$, keeping the nucleon number density constant. Note that this cooling time scale is much larger than the time scale $\tau_{\text {relax }}$ for relaxation of the system, which is estimated as $\tau_{\text {relax }} \sim$ (length scale of the inhomogeneity)/(sound velocity) $\sim 10 \mathrm{fm} / 0.1 c=100 \mathrm{fm} / c$. In the cooling process, we mainly used the frictional relaxation method, which is given by the QMD equations of motion plus small friction terms: $\dot{\mathbf{R}}_{i}=\partial \mathscr{H} / \partial \mathbf{P}_{i}-\xi_{R} \partial \mathscr{H} / \partial \mathbf{R}_{i}, \dot{\mathbf{P}}_{i}=-\partial \mathscr{H} / \partial \mathbf{R}_{i}-\xi_{P} \partial \mathscr{H} / \partial \mathbf{P}_{i}$, where $\mathscr{H}$ is the QMD Hamiltonian, $\mathbf{R}_{i}$ and $\mathbf{P}_{i}$ are the position and momentum of nucleon $i$, respectively, and $\xi_{R}, \xi_{P}>0$ are the frictional coefficients.

The resulting nucleon distributions of cold matter at $x=0.5$ are shown in Fig. 1 We see from these figures that the phases with rod-like and slab-like nuclei, cylindrical and spherical bubbles, in addition to the phase with spherical nuclei are reproduced.

1 This calculation is based on the linearized Thomas-Fermi approximation and thus the true value of $\delta E_{e} / n_{\mathrm{N}}$ would be larger than this value. 

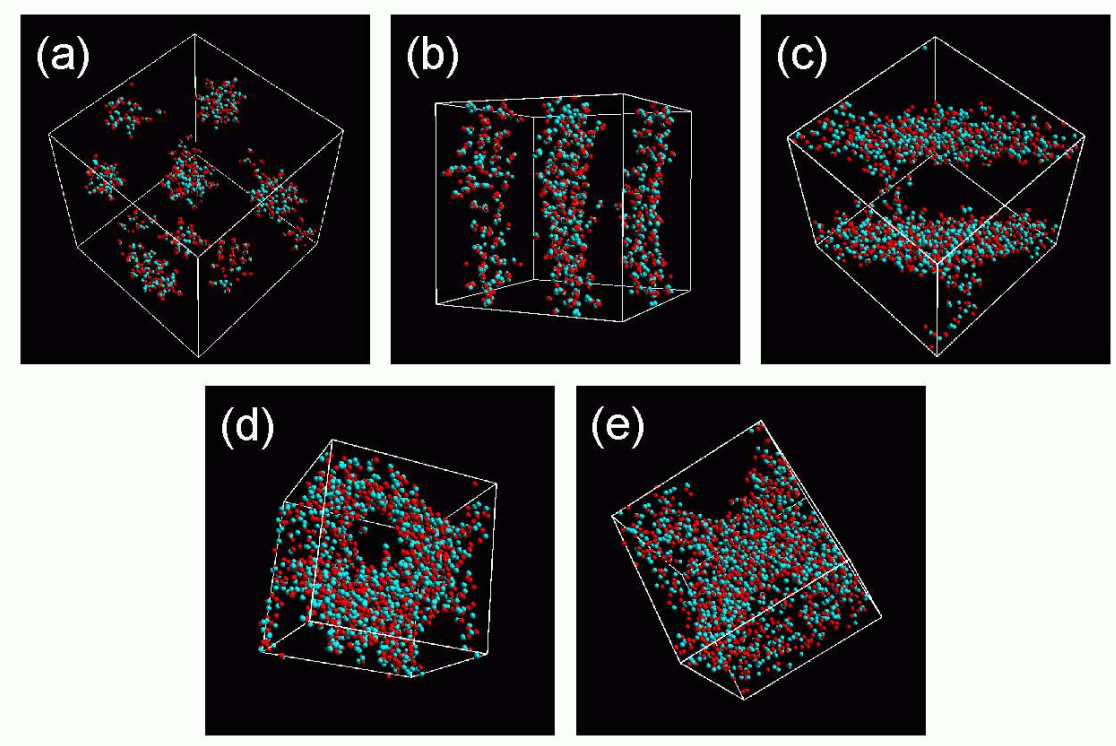

FIGURE 1. Nucleon distributions of the pasta phases in cold matter at $x=0.5$; (a) sphere phase, $0.1 \rho_{0}$; (b) cylinder phase, $0.225 \rho_{0}$; (c) slab phase, $0.4 \rho_{0}$; (d) cylindrical hole phase, $0.5 \rho_{0}$; (e) spherical hole phase, $0.6 \rho_{0}$. The red (darker) particles represent protons and the green (brighter) ones neutrons. Taken from Ref. [12].

The above simulations show that the pasta phases can be formed dynamically from hot uniform matter within a time scale $\sim O\left(10^{3}-10^{4}\right) \mathrm{fm} / c$.

In Fig. 2, we show snapshots of the nucleon distributions for $\rho=0.225 \rho_{0}$ at $T=1,2$ and $3 \mathrm{MeV}$. This density corresponds to the phase with rod-like nuclei at $T=0$. We have observed the following qualitative features: at $T \simeq 1.5-2 \mathrm{MeV}$ the number of evaporated nucleons becomes significant; at $T \gtrsim 3 \mathrm{MeV}$, nuclei almost melt and the nuclear surface is hard to identify.

The phase diagram for $x=0.5$ is plotted in Fig. 3. The critical temperature of this model is $\gtrsim 6 \mathrm{MeV}$. In the region below the dotted lines at $T \lesssim 3 \mathrm{MeV}$, where we can identify the nuclear surface, we have obtained the pasta phases in the same sequence as in the earlier works: from lower densities, spherical nuclei [region (a)], rod-like nuclei [region (b)], slab-like nuclei [region (d)], cylindrical holes [region (f)], and spherical holes [region $(\mathrm{g})$ ]. In addition to these pasta phases, structures with multiply connected nuclear and bubble regions (i.e., sponge-like structure) such as branching rod-like nuclei, perforated slabs and branching bubbles, etc., have been obtained in the regions (c) and (e). Further study using a larger system is necessary to conclude the existence of these sponge-like phases (see also Refs. [1, 25, 26]).

\section{Structural Transitions between the Pasta Phases}

In Ref. [14], we demonstrated the second point mentioned at the beginning of this article. We performed QMD simulations of the compression of dense matter and observed 
(a) $\mathrm{T}=1 \mathrm{MeV}$
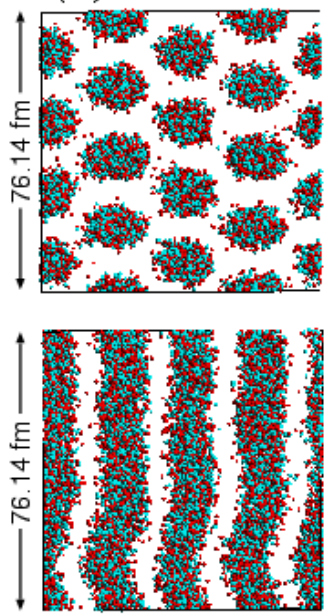

(b) $\mathrm{T}=2 \mathrm{MeV}$
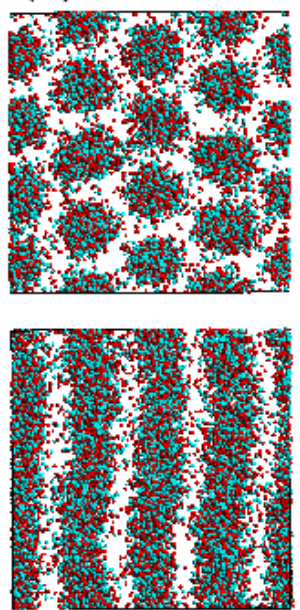

(c) $\mathrm{T}=3 \mathrm{MeV}$
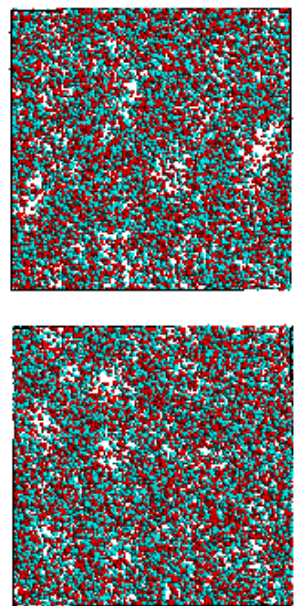

FIGURE 2. Nucleon distributions for $x=0.5, \rho=0.225 \rho_{0}$ at temperatures of 1,2 and $3 \mathrm{MeV}$. The total number of nucleons $N=16384$ and the box size $L_{\text {box }}=76.14 \mathrm{fm}$. The upper panels show top views along the axis of the cylindrical nuclei at $T=0$, and the lower ones show side views. Protons are represented by the red (darker) particles, and neutrons by the green (lighter) ones. Taken from Ref. [13].

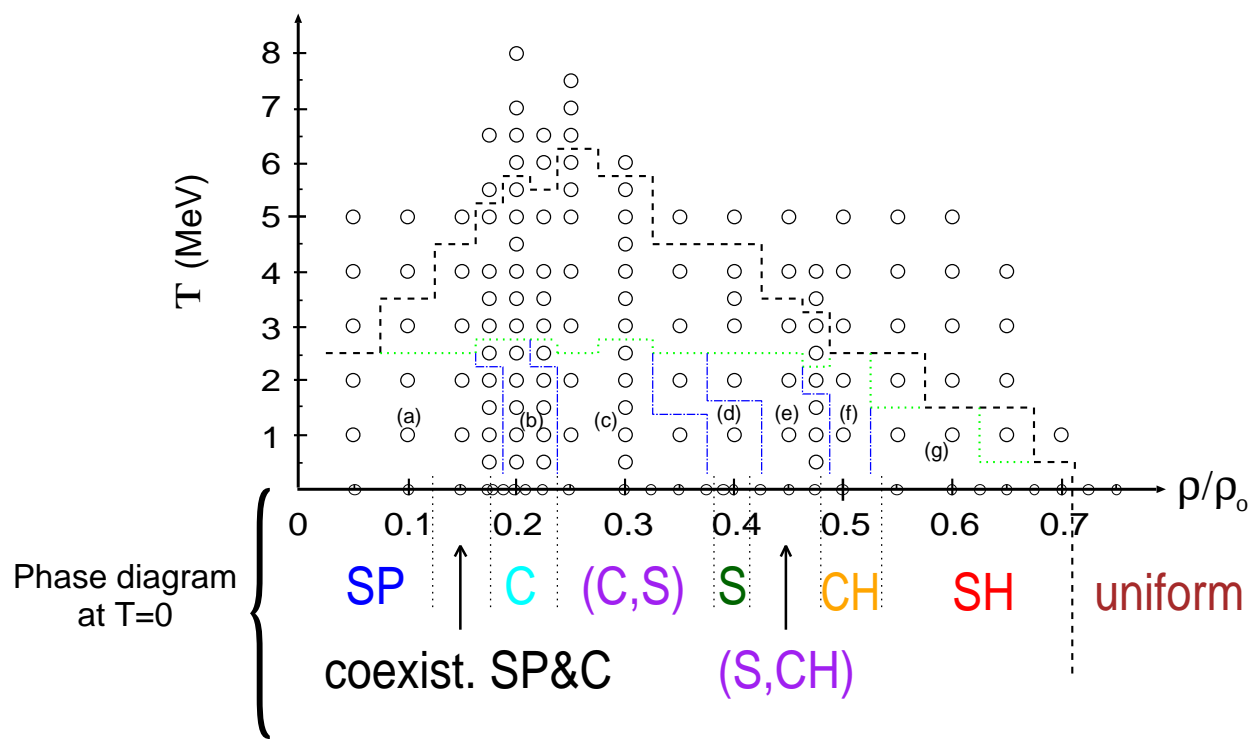

FIGURE 3. Phase diagram of matter at $x=0.5$ plotted in the $\rho-T$ plane. The dashed and the dotted lines on the diagram show the phase separation line and the limit below which the nuclear surface can be identified, respectively. The dash-dotted lines are the phase boundaries between the different nuclear shapes. The symbols SP, C, S, CH, SH, U stand for nuclear shapes, i.e., sphere, cylinder, slab, cylindrical hole, spherical hole and uniform, respectively. The parentheses $(\mathrm{A}, \mathrm{B})$ denote an intermediate phase between A and B-phases with a multiply connected structure. Simulations have been carried out at points denoted by circles. Adapted from Ref. [13]. 

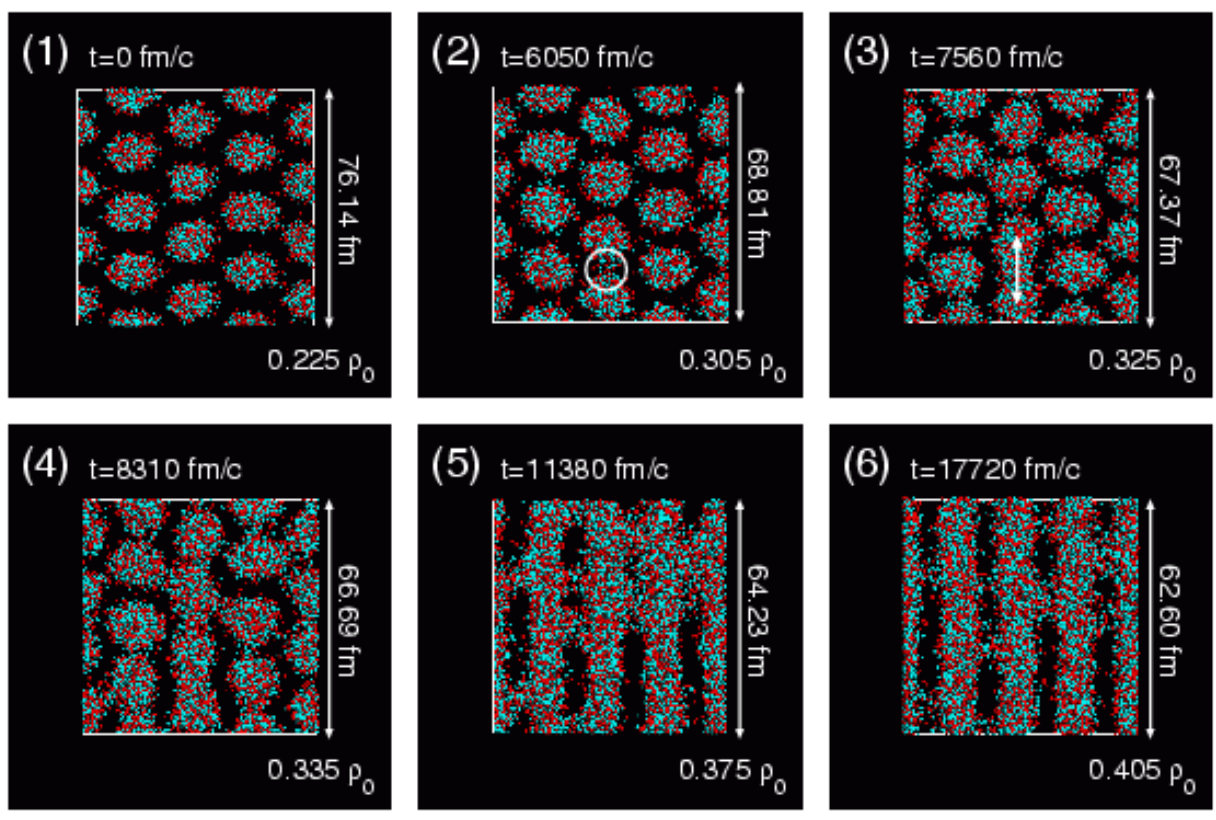

FIGURE 4. Snapshots of the transition process from the phase with rod-like nuclei to the phase with slab-like nuclei. The red (darker) particles show protons and the green (lighter) ones neutrons. The box size is rescaled to be equal in this figure. Adapted from Ref. [14].

the transitions from rod-like nuclei to slab-like ones and from slab-like nuclei to rod-like bubbles.

The initial conditions of the simulations are samples of the phase with rod-like nuclei $\left(\rho=0.225 \rho_{0}\right)$ and of the phase with slab-like nuclei $\left(\rho=0.4 \rho_{0}\right)$ with 16384 nucleons at $x=0.5$ and $T \simeq 1 \mathrm{MeV}$. We adiabatically compressed these samples by increasing the density to the value corresponding to the next pasta phase taking $O\left(10^{4}\right) \mathrm{fm} / c$. This time scale is much larger than the typical time scale of the deformation and structural transition of nuclei (e.g., that of nuclear fission is $\sim 1000 \mathrm{fm} / c$ ). Therefore, the compression in the simulations is adiabatic with respect to the change of the nuclear structure, so that the dynamics of the structural transition of nuclei observed in the simulations is physically meaningful, and is essentially independent of the compression rate, etc.

The resulting time evolution of the nucleon distribution is shown in Figs. 4 and 5, In Fig. 4] we see that the phase with slab-like nuclei is finally formed [Fig. 4-(6)] from the phase with rod-like nuclei [Fig. 4-(1)]. We note that the transition is triggered by thermal fluctuations, not by the fission instability: when the internuclear spacing becomes small enough and once some pair of neighboring rod-like nuclei touch due to the thermal fluctuations, they fuse [Figs. 4-(2) and 4-(3)]. Then such connected pairs of rod-like nuclei further touch and fuse with neighboring nuclei in the same lattice plane like a chain reaction [Fig. 4-(4)]; the time scale of the each fusion process is $O\left(10^{2}\right) \mathrm{fm} / c$.

The transition from the phase with slab-like nuclei to the phase with cylindrical holes is shown in Fig. 5] When the internuclear spacing decreases enough, neighboring slablike nuclei touch due to the thermal fluctuation as in the above case. Once nuclei begin to 

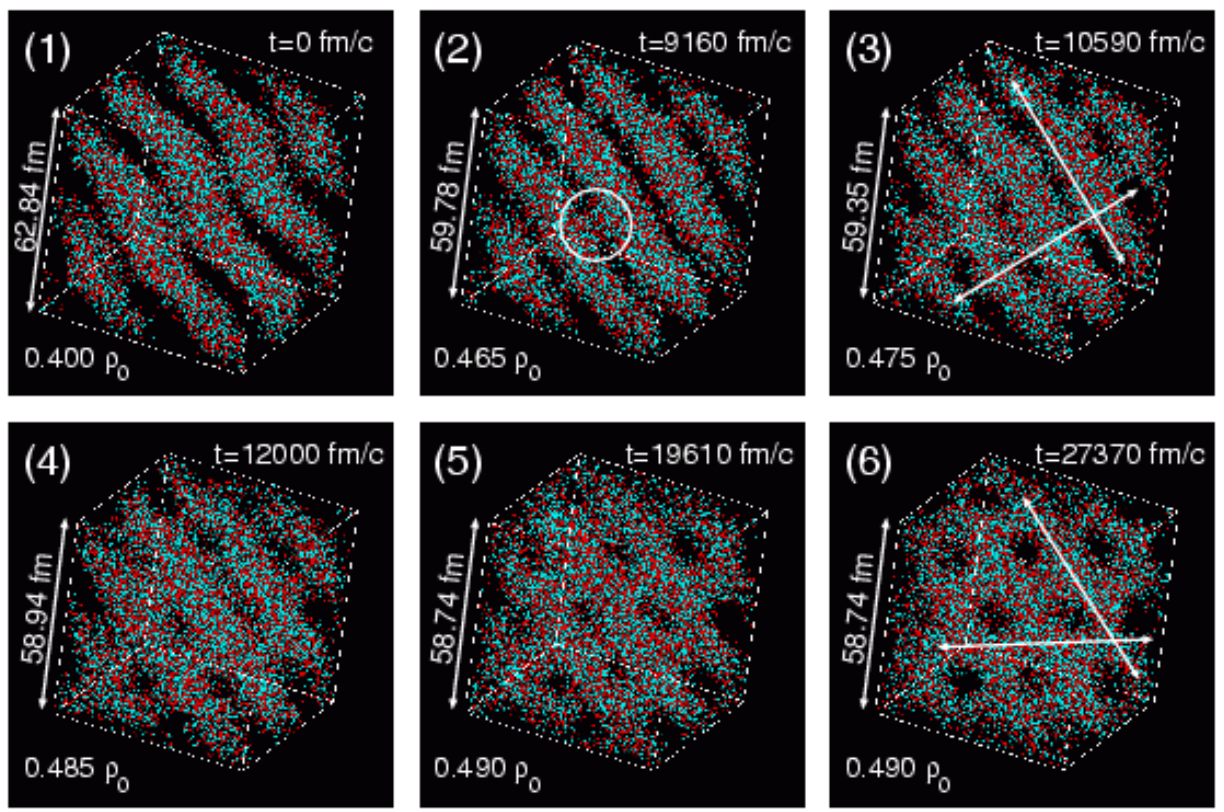

FIGURE 5. The same as Fig. [4 for the transition from the phase with slab-like nuclei to the phase with cylindrical holes (the box size is not rescaled in this figure). Adapted from Ref. [14].

touch [Fig. 5-(2)], bridges between the slabs are formed at many places on a time scale of $O\left(10^{2}\right) \mathrm{fm} / c$. Initially, the bridges cross the slabs almost orthogonally [Fig. 55-(3)]. Nucleons in the slabs continuously flow into the bridges and the bridges become wider and merge together to form cylindrical holes. Then the cylindrical holes finally relax into a triangular lattice [Fig. 5-(6)].

\section{SUMMARY AND FUTURE PROSPECTS}

Using QMD, we have shown the following two things: formation of the pasta phases in neutron star crusts by cooling and formation of rod-like bubbles from slab-like nuclei and that of slab-like nuclei from rod-like ones in supernova cores by compression of matter. In closing, we list important issues to be clarified in the future.

1. Formation of the pasta phases by compression [27] A remaining problem is a transition from a bcc lattice of spherical nuclei to a triangular lattice of rod-like nuclei induced by compression. If this process is confirmed, existence of the pasta phases in supernova cores will be almost established.

2. Effects of uncertainties of nuclear forces \& properties of nuclei

We employed a specific nuclear force. Although we have confirmed that this nuclear interaction yields reasonable values for the surface energy and the proton and neutron chemical potentials in neutron matter, etc. [12], in addition to reproducing saturation properties [18], further systematic survey is needed to understand effects of uncertainties in nuclear properties on the pasta phases [28]. Especially, uncer- 
tainties in the surface properties are the most important elements to be examined.

3. Detailed study of astrophysical consequences

If formation of the pasta phases in actual astrophysical situations is established, quantitative discussion about effects of the pasta phases on astrophysical phenomena will be increasingly important. As we have stressed previously [10, 12], effects of the pasta structure on coherent scattering of neutrinos in the stage of the neutrino trapping is an interesting problem [24, 13, 28, 29].

\section{ACKNOWLEDGMENTS}

The author is grateful to Chris Pethick for helpful comments. The research reported in this article grew out of collaborations with H. Sonoda, K. Sato, T. Maruyama, K. Yasuoka, T. Ebisuzaki, and K. Iida. Further research currently in progress is performed using the RIKEN Super Combined Cluster System with MDGRAPE-2. This work was supported in part by a JSPS Postdoctoral Fellowship for Research Abroad, by the Nishina Memorial Foundation, by the Japan Society for the Promotion of Science, by the Ministry of Education, Culture, Sports, Science and Technology through Research Grant No. 14-7939, and by RIKEN through Research Grant No. J130026.

\section{REFERENCES}

1. C. J. Pethick and D. G. Ravenhall, Annu. Rev. Nucl. Part. Sci. 45, 429 (1995).

2. N. Chamel, contribution to this volume.

3. D. G. Ravenhall, C. J. Pethick, and J. R. Wilson, Phys. Rev. Lett. 50, 2066 (1983).

4. M. Hashimoto, H. Seki, and M. Yamada, Prog. Theor. Phys. 71, 320 (1984).

5. R. D. Williams and S. E. Koonin, Nucl. Phys. A435, 844 (1985).

6. M. Lassaut et al., Astron. Astrophys. 183, L3 (1987).

7. C. P. Lorenz, D. G. Ravenhall, and C. J. Pethick, Phys. Rev. Lett. 70, 379 (1993).

8. K. Oyamatsu, Nucl. Phys. A561, 431 (1993).

9. K. Sumiyoshi, K. Oyamatsu, and H. Toki, Nucl. Phys. A595, 327 (1995).

10. G. Watanabe, K. Iida, and K. Sato, Nucl. Phys. A676, 455 (2000); ibid. A687, 512 (2001); Erratum, ibid. A726, 357 (2003).

11. J. Aichelin and H. Stöcker, Phys. Lett. B176, 14 (1986); J. Aichelin, Phys. Rep. 202, 233 (1991).

12. G. Watanabe et al., Phys. Rev. C 66, 012801(R) (2002); ibid. 68, 035806 (2003).

13. G. Watanabe et al., Phys. Rev. C 69, 055805 (2004).

14. G. Watanabe et al., Phys. Rev. Lett. 94, 031101 (2005).

15. K. Oyamatsu and M. Yamada, Nucl. Phys. A578 (1994) 181.

16. P. Magierski and P. -H. Heenen, Phys. Rev. C 65 (2002) 045804.

17. N. Chamel, Nucl. Phys. A747, 109 (2005).

18. T. Maruyama et al., Phys. Rev. C 57, 655 (1998).

19. T. Kido et al., Nucl. Phys. A663 \& 664, 877c (2000).

20. K. Niita, in the Proceedings of the Third Simposium on "Simulation of Hadronic Many-body System”, A. Iwamoto et al., Eds., JAERI-conf. 96-009, 22 (1996) (in Japanese).

21. G. Watanabe and K. Iida, Phys. Rev. C 68, 045801 (2003).

22. T. Maruyama et al., Phys. Rev. C 72, 015802 (2005).

23. G. Baym, H. A. Bethe, and C. J. Pethick, Nucl. Phys. A175, 225 (1971).

24. C. J. Horowitz et al., Phys. Rev. C 70, 065806 (2004).

25. G. Watanabe and H. Sonoda, to appear in "Soft Condensed Matter: New Research", ed. K. I. Dillon (cond-mat/0502515). 
26. M. Matsuzaki, Phys. Rev. C 73, 028801 (2006).

27. G. Watanabe et al., in preparation.

28. H. Sonoda et al., in preparation.

29. H. Sonoda, contribution to this volume. 